\title{
PATOGENICIDAD DE TRES CEPAS DE HONGOS ENTOMOPATÓGENOS A ADULTOS DE ANASTREPHA OBLIQUA (MACQUART) (DIPTERA: TEPHRITIDAE) EN CONDICIONES DE LABORATORIO
}

\author{
Noé Hernández díaz-Ordaz1,2, Nelson Pérez² y Jorge Toledo 1 \\ 1Departamento de Entomología Tropical. El Colegio de la Frontera Sur. Carretera Antiguo Aeropuerto \\ Km. 2.5. Apdo. Postal 36, Tapachula, Chiapas. C. P. 30700. MÉXICO. \\ 2Facultad de Ciencias Agrícolas. Universidad Autónoma de Chiapas. Car. Costera entronque a \\ Huehuetán. C. P. 30660 Huehuetán, Chiapas, MÉXICO.
}

Hernández Díaz-Ordaz, N., N. Pérez \& J. Toledo. 2010. Patogenicidad de Tres Cepas de Hongos Entomopatógenos a Adultos de Anastrepha obliqua (Macquart) (Diptera: Tephritidae) en Condiciones de Laboratorio. Acta Zool. Mex. (n. s.), 26(3): 481-494.

RESUMEN. Buscando alternativas biorracionales para el control de moscas de la fruta, se evaluó la actividad de dos cepas del hongo entomopatógeno Beauveria bassiana $\left(\mathrm{Bb}_{26}\right.$ y $\left.\mathrm{Bb}_{\mathrm{JLSV}}\right)$ y una de Metarhizium anisopliae ( $\mathrm{Ma}_{\mathrm{CENGICANA}}$ ) contra adultos de Anastrepha obliqua en laboratorio. La cepa $\mathrm{Bb}_{26}$ fue la que ocasionó mayor infección (99.8\%), seguida por la cepa $\mathrm{Bb}_{\mathrm{JLSV}}(93.5 \%)$, y de la cepa $\mathrm{Ma}_{\text {CENGICAÑa }}(89.8 \%)$. Tanto hembras como machos fueron susceptibles a la infección por hongos de las cepas evaluadas, observándose una mortalidad similar para adultos de ambos sexos. La cepa

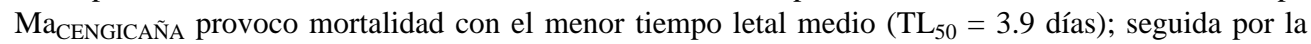
cepa $\mathrm{Bb}_{26}$ (5.3 días) y la cepa $\mathrm{Bb}_{\mathrm{JLSV}}$ (6.4 días). El tiempo letal medio promedio para el conjunto de las tres cepas fue de 5.2 días. Los resultados indican que la cepa más patogénica contra adultos fue la $\mathrm{Bb}_{26}$, con una concentración letal media $\left(\mathrm{CL}_{50}\right)$ de $3.8 \times 10^{6}$ conidios $\mathrm{ml}^{-1}$, seguida por la cepa Ma $\mathrm{CENGICAÑA}_{\mathrm{A}}$ con una $\mathrm{CL}_{50}$ equivalente a $4.8 \times 10^{6}$ conidios $\mathrm{ml}^{-1}$ y la cepa $\mathrm{Bb}_{\mathrm{JLSV}}$ con una $\mathrm{CL}_{50}$ equivalente a $8.8 \mathrm{x}$ $10^{6}$ conidios $\mathrm{ml}^{-1}$. La cepa $\mathrm{Bb}_{26}$ fue la que produjo mayor cantidad de conidios, ya que las moscas infectadas que fueron tratadas con una concentración de $1.52 \times 10^{8}$ conidios ml-1 registraron $77.9 \%$ de esporulación; seguida de las cepas $\mathrm{Ma}_{\text {CENGICAÑA }}\left(1.50 \times 10^{8}\right.$ conidios $\left.\mathrm{ml}^{-1}\right)$ y $\mathrm{Bb}_{\mathrm{JLSV}}\left(1.76 \times 10^{8}\right.$ conidios $\mathrm{ml}^{-1}$ ) que registraron 73.8 y $72.6 \%$ de esporulación, respectivamente. La esporulación de los cadáveres infestados registró una correlación positiva entre la concentración aplicada y la producción de conidios $\left(\mathrm{R}^{2}=0.91, \mathrm{Y}=1.63+0.14 \mathrm{x}\right)$, lo cual indicó que conforme se incrementó la concentración para tratar las moscas, fue mayor la producción de conidios en las moscas infectadas.

Palabras clave: Control microbiano, entomopatógeno, Anastrepha obliqua, moscas de la fruta.

Hernández Díaz-Ordaz, N., N. Pérez \& J. Toledo. 2010. Pathogenecity of three strains of entomopathogenic fungus on Anastrepha obliqua adults (Macquart) (Diptera: Tephritidae) under laboratory conditions. Acta Zool. Mex. (n. s.), 26(3): 481-494.

Recibido: 24/02/2009; aceptado: 17/06/2010. 


\begin{abstract}
In order to develop biorrational fruit fly control methods, the activity of two strains of Beauveria bassiana $\left(\mathrm{Bb}_{26}\right.$ and $\left.\mathrm{Bb}_{\mathrm{JLSV}}\right)$ and a strain of Metarhizium anisopliae $\left(\mathrm{Ma}_{\mathrm{CENGICAN} \mathrm{A}}\right)$ against adults of Anastrepha obliqua was evaluated in the laboratory. The $\mathrm{Bb}_{26}$ strain caused the highest infection rate $\left(99.8 \%\right.$ ), followed by the $\mathrm{Bb}_{\text {JLSV }}$ strain $\left(93.5 \%\right.$ ), and the Ma $\mathrm{M}_{\text {CENGICAÑA }}$ strain which caused the lowest infection (89.8\%). Both males and females were susceptible to the strains tested, with a similar mortality in both sexes. The median lethal time $\left(\mathrm{LT}_{50}\right)$ was 3.9, 5.3 and 6.4 days for $\mathrm{Ma}_{\text {CENGICAÑA, }} \mathrm{Bb}_{26}$ and $\mathrm{Bb}_{\text {JLSV }}$, respectively, with an average median lethal time for the three strains of 5.2 days. Results indicate that the most pathogenic strain against adults of $A$. obliqua was $\mathrm{Bb}_{26}$, with a median lethal concentration $\left(\mathrm{LC}_{50}\right.$ ) equivalent to $3.8 \times 10^{6}$ conidia $\mathrm{ml}^{-1}$, followed by the Ma $\mathrm{CENGICANA}_{\mathrm{A}}$ strain equivalent to $4.8 \times 10^{6}$ conidia $\mathrm{ml}^{-1}$ and the $\mathrm{Bb}_{\mathrm{JLSV}}$ strain with an equivalent to $8.8 \times 10^{6}$ conidia $\mathrm{ml}^{-1}$. The $\mathrm{Bb}_{26}$ strain produced the highest sporulation, with a concentration of $1.52 \times 10^{8}$ conidia ml-1, and a sporulation of $77.9 \%$ of infected adults; followed by the Ma ${ }_{\text {CENGICAÑA }}$ strain $\left(1.50 \times 10^{8}\right.$ conidia $\left.\mathrm{ml}^{-1}\right)$ and $\mathrm{Bb}_{\mathrm{JLSV}}\left(1.76 \times 10^{8}\right.$ conidia $\left.\mathrm{ml}^{-1}\right)$ with 73.8 and $72.6 \%$ of sporulation, respectively. Sporulation of infected cadavers and inoculum concentration showed a positive correlation between the applied concentration and the production of conidia by flies $\left(\mathrm{R}^{2}=0.91, \mathrm{Y}=1.63+0.14 \mathrm{x}\right)$, which suggested that when increasing the concentration of inoculum, the production of conidia tended to increase.
\end{abstract}

Key words: Microbial control, entomopathogen, Anastrepha obliqua, fruit flies.

\title{
INTRODUCCIÓN
}

En México, el mango es atacado por Anastrepha obliqua (Macquart) y debido al alto valor comercial que posee este cultivo, esta especie es considerada como la segunda en importancia económica en el país. Ante esta situación, se inicio un programa de manejo a gran escala con un enfoque integral (Reyes et al. 2000). Sin embargo, para la supresión de adultos se siguen utilizando insecticidas convencionales, generando problemas sociales, ambientales y la posibilidad de que los insectos desarrollen resistencia a estos productos (Aluja 1994, Magaña et al. 2007, Troestchler 1984), por lo que el uso de hongos entomopatógenos para el control de esta plaga tiene potencial.

La patogenicidad de las diferentes cepas de hongos entomopatógenos contra moscas de la fruta (Tephritidae) ha sido poco estudiada y los resultados obtenidos son promisorios, por lo que se requiere explorar nuevas cepas (Quesada-Moraga et al. 2006, Muñoz et al. 2009, Sookar et al. 2008). La caracterización patogénica se ha realizado contra las distintas fases biológicas del insecto, en Ceratitis capitata (Wied.), algunas cepas de Metarhizium anisopliae (Metchnikoff) Sorokin y de Beauveria bassiana (Balsamo) Vuillemin, son activas contra pupas y adultos, pero no contra larvas (García et al. 1984, Espín-García et al. 1989). Con Anastrepha ludens (Loew), varias cepas de B. bassiana y de $M$. anisopliae registraron baja actividad biológica en larvas (Campos 2000, De la Rosa et al. 2002). Estos mismos autores reportan que las cepas de $M$. anisopliae tienen baja actividad y las cepas de $B$. bassiana no son activas contra pupas. Sin embargo hay contraste, ya que algunas cepas de hongos son patogénicas a esta fase del insecto (Lezama-Gutiérrez et al. 2000, Quesada-Moraga et al. 2006). 
Para las dos especies de hongos se ha aislado un mayor número de cepas activas contra adultos de varias especies de moscas de la fruta (Campos 2000, LezamaGutiérrez et al. 2000, De la Rosa et al. 2002, Dimbi et al. 2003, Sookar et al. 2008). Sin embargo, la actividad biológica entre cepas puede variar, como se observó en adultos de $C$. capitata tratados con algunas cepas de B. bassiana (Quesada-Moraga et al. 2006, Muñoz et al. 2009, Sookar et al. 2008). También el sexo del hospedero puede afectar la actividad, ya que en algunas especies los machos son más susceptibles (Campos 2000, De la Rosa et al. 2002).

La actividad biológica del patógeno demostrada para una especie puede no resultar efectiva contra otra especie cercana, como se observó con una cepa de $M$. anisopliae evaluada contra adultos de C. capitata y A. obliqua, resultando la primera especie más susceptible (Herrera \& Mata 2006). También el origen del hospedero puede influir, en adultos silvestres de A. ludens tratados con una cepa de B. bassiana, se obtuvo $>60 \%$ de mortalidad, y con dos cepas de $M$. anisopliae se registró $\sim 83 \%$ de infección, pero la actividad fue menor en insectos procedentes de un sistema de cría (Arévalo-Niño et al. 2006).

Considerando que la exploración de alternativas biorracionales como el uso de hongos entomopatógenos, para incorporarlos a los esquemas de manejo de dicha plaga, cómo estrategias con menor impacto ambiental, es prioritaria. Este trabajo tuvo como objetivo evaluar la patogenicidad de tres cepas de hongos entomopatógenos (una de $M$. anisopliae y dos de B. bassiana), contra adultos de $A$. obliqua en condiciones de laboratorio.

\section{MATERIAL Y MÉTODOS}

Sitio de estudio y condiciones ambientales. Esta investigación se realizó en las instalaciones de El Colegio de la Frontera Sur (ECOSUR), Unidad Tapachula, en el Laboratorio de Patología de Insectos, de la línea de investigación Manejo Integrado de Plagas en colaboración con la línea de Ecología de Poblaciones de Moscas de la Fruta, en Tapachula, Chiapas, México. Las condiciones ambientales que prevalecieron durante el desarrollo del estudio fueron: temperatura de $26 \pm 1{ }^{\circ} \mathrm{C}, \mathrm{H}$.R. de $70 \pm 5 \%$, y fotoperiodo de 12: $12 \mathrm{~L}: \mathrm{O}$.

Material biológico. Las cepas de los hongos entomopatógenos evaluadas fueron, una de Metarhizium anisopliae (Ma $\mathrm{M}_{\mathrm{CENGICAÑA}}$, proporcionada por el Centro Guatemalteco de Investigación en Caña de Azúcar), seleccionada debido a que se produce en cantidades grandes para el control exitoso de mosca pinta (Aeneolamia sp.) en cultivo de caña de azúcar y dos cepas de Beauveria bassiana (cepas $\mathrm{Bb}_{\mathrm{JLSV}} \mathrm{y}$ $\mathrm{Bb}_{26}$ ), que fueron seleccionadas debido a que la primera se produce a escala comercial en un laboratorio del Soconusco, Chiapas, y previamente se demostró que es patógena a adultos de A. ludens, y para la segunda cepa se demostró que fue 
patógena a adultos de A. ludens y C. capitata (De la Rosa et al. 2002, Muñoz et al. 2009). Para la reproducción de cada cepa de hongo se utilizó el medio de cultivo Agar Dextrosa Saboraud (ADS), que se preparó con una cantidad de $65 \mathrm{~g}$ de agar disuelto en un litro de agua destilada $+5 \mathrm{~g}$ de extracto de levadura. El medio de cultivo fue vaciado en tubos de ensayo que posteriormente se esterilizaron en autoclave a $120^{\circ} \mathrm{C}$ por 15 minutos $\left(15 \mathrm{lb} / \mathrm{pulg}^{-2}\right)$. El agar utilizado para las cajas de Petri, fue esterilizado antes de vaciarse junto con los tubos de ensayo. La siembra de cada cepa se realizó en el interior de una campana de flujo laminar, depositando con un asa bacteriológica estéril los conidios en tubos de ensayo y en las cajas de Petri. Posterior a la siembra los tubos de ensayo y las cajas de Petri se incubaron a $27 \pm 2^{\circ} \mathrm{C}$, a una humedad relativa de $80 \pm 5 \%$ bajo un fotoperiodo de $12: 12$ horas luzoscuridad, durante 15 días. Después de este tiempo, los conidios fueron cosechados realizando un raspado de la superficie del cultivo con un asa bacteriológica estéril.

Debido a que estudios previos indicaron que estas cepas no son activas contra larvas y pupas, y el objetivo es tener al menos una de ellas para utilizarse contra adultos, se tomo la decisión de evaluarse solamente contra adultos. Se utilizaron adultos estériles de A. obliqua, irradiados en estado de pupa con Cobalto 60 a una dosis de $80 \mathrm{~Gy}$, proporcionados por el laboratorio del Programa Moscafrut (SAGARPA-IICA), ubicado en Metapa de Domínguez, Chiapas, México, en donde las larvas se alimentan y se desarrollan sobre una dieta artificial de acuerdo a la metodología y condiciones ambientales requeridas durante el proceso (Artiaga-López et al. 2004). Los bioensayos fueron realizados con adultos de 6 a 9 días de edad.

Viabilidad de los conidios. La viabilidad de los conidios de cada cosecha se determinó utilizando el método de microcultivos hechos con ADS. Las muestras para los microcultivos fue preparada utilizando una pequeña cantidad de conidios que se colocó en tubos de vidrio con tapón de rosca con $3 \mathrm{~mL}$ de agua destilada estéril + glicerina como dispersante-adherente (Jiménez 1992). La solución se homogenizó con un vortex durante 10 minutos y después se colocaron alícuotas de 20 microlitros sobre un área de $1.5 \times 2.0 \mathrm{~cm}$ cubierta con una película de ADS en cada portaobjeto. Los portaobjetos fueron colocados en cajas de Petri que contenían torundas de algodón humedecido para promover la germinación de los conidios y 18 horas después de inoculados se hicieron observaciones bajo un microscopio compuesto, considerando como conidio germinado el que presentó un crecimiento del tubo germinativo igual o mayor a 10 micras de longitud. Una cepa se consideró viable para realizar los bioensayos, cuando registró $\geq 90 \%$ de conidios germinados, de un total de cinco repeticiones por cosecha para cada cepa.

Patogenicidad hacia adultos. Para esta prueba se hizo una dilución que se preparó para cada cepa mediante una solución fungosa al $1 \%$ utilizando $0.3 \mathrm{~g}$ de conidios 
diluidos en $30 \mathrm{~mL}$ de agua destilada estéril $+1 \mathrm{~mL}$ de dispersante-adherente, homogenizando la muestra durante 30 minutos con un agitador magnético. Las moscas fueron separadas en grupos de 15 parejas (relación de sexo 1:1) y se colocaron en tubos de ensayo, a los que se les adicionaron $3 \mathrm{~mL}$ de la solución fungosa correspondiente, considerando a cada cepa como un tratamiento y por cada tratamiento se realizaron tres repeticiones. Las moscas del testigo fueron tratadas solamente con agua destilada estéril + adherente. De acuerdo con la metodología desarrollada por De la Rosa et al. (2002), después de que las moscas fueron tratadas, se colocaron en una caja de Petri que contenía papel absorbente para eliminar el exceso de humedad y posteriormente fueron transferidas a jaulas de vidrio de $30 \mathrm{x}$ 30 x $30 \mathrm{~cm}$, acondicionada en uno de sus lados con una malla de tela sintética para facilitar el manejo. Cada jaula contenía en su interior una mezcla de proteína hidrolizada enzimáticamente (MP Biomedical, Irvine, CA) + azúcar (proporción 1:3) como fuente de alimento y el agua fue proporcionada en algodones a punto de saturación colocados sobre tapas de cajas de Petri de plástico.

Las jaulas se colocaron en un cuarto bajo condiciones controladas de temperatura $\left(27 \pm 2{ }^{\circ} \mathrm{C}\right)$, humedad relativa $(80 \pm 5 \%)$ y un fotoperiodo (12:12 h luz-oscuridad). Diariamente se llevó un registro de la mortalidad de los insectos durante 10 días. Las moscas muertas se colocaron en cámara húmeda (tapa de caja de Petri + papel filtro + algodón húmedo) para promover el crecimiento del micelio y confirmar la muerte por infección del hongo.

Tiempo letal medio. El tiempo letal medio $\left(\mathrm{TL}_{50}\right)$ para cada cepa se determinó en adultos de acuerdo a la metodología previamente descrita para la prueba de patogenicidad. Se preparó una solución madre al 1\% homogenizándose durante 30 minutos, posteriormente se colocaron las moscas en tubos de ensayo y con una pipeta se depositaron $3 \mathrm{~mL}$ de la solución fungosa para tratarlas. Se realizaron tres repeticiones con 30 moscas (proporción de sexos de 1:1). Por cada repetición y por cada cepa se manejó un testigo con la misma cantidad de insectos.

Después que las moscas fueron inoculadas se colocaron en papel absorbente para eliminar el exceso de agua y se transfirieron a las jaulas de vidrio manteniéndose bajo condiciones controladas de temperatura y humedad. Se llevó un registro diario de la mortalidad a partir del segundo día después de la inoculación. Los insectos muertos se colocaron en cámaras húmedas (previamente descritas) para promover el desarrollo del hongo y corroborar la infección.

Concentración letal media. Para determinar la concentración letal media $\left(\mathrm{CL}_{50}\right)$, que cumplieran con los requisitos estadísticos establecidos para este tipo de estudios (Ibarra \& Federici 1987), se evaluó una serie de concentraciones intermedias preparadas por dilución a partir de la solución fungosa al 1.0\% de cada cepa (Cuadro 
1), que fueron determinadas a partir de las concentraciones que infectaron al menor y mayor número de moscas en el estudio de patogenicidad. Para cada cepa se realizaron tres bioensayos independientes, para cada bioensayo se hicieron tres repeticiones por concentración, y para cada concentración se trataron 15 parejas de insectos (relación de sexo 1:1). Como testigo, se utilizó la misma cantidad de insectos, tratados solamente con agua destilada estéril + adherente. El manejo de las moscas tratadas, el registro de la mortalidad y la verificación de la infección se realizó como se describió previamente, considerando que los bioensayos eran aceptables cuando la mortalidad en los insectos del testigo fue igual o menor a $10 \%$.

Cuadro 1. Concentraciones de las cepas de los hongos entomopatógenos evaluadas contra adultos de A. obliqua mediante el método de impregnación.

\begin{tabular}{|c|c|c|c|}
\hline \multirow[t]{2}{*}{ Concentración (\%) } & \multicolumn{3}{|c|}{ Conidios ml-1 } \\
\hline & $\left(\mathrm{Bb}_{26}\right)$ & Conidios ml-1 $\left(\mathrm{Bb}_{\mathrm{JLSV}}\right)$ & Conidios $\mathrm{ml}^{-1}\left(\mathrm{Ma}_{\text {CENGICAÑA }}\right)$ \\
\hline 1.0 & $1.52 \times 10^{8}$ & $1.76 \times 10^{8}$ & $1.50 \times 10^{8}$ \\
\hline 0.5 & $7.6 \times 10^{7}$ & $8.8 \times 10^{7}$ & $7.5 \times 10^{7}$ \\
\hline 0.1 & $1.52 \times 10^{7}$ & $1.76 \times 10^{7}$ & $1.50 \times 10^{7}$ \\
\hline 0.05 & $7.6 \times 10^{6}$ & $8.8 \times 10^{6}$ & $7.5 \times 10^{6}$ \\
\hline 0.01 & $1.52 \times 10^{6}$ & $1.76 \times 10^{6}$ & $1.50 \times 10^{6}$ \\
\hline 0.005 & $7.6 \times 10^{5}$ & $8.8 \times 10^{5}$ & $7.5 \times 10^{5}$ \\
\hline 0.001 & $1.52 \times 10^{5}$ & $1.76 \times 10^{5}$ & $1.50 \times 10^{5}$ \\
\hline
\end{tabular}

Producción y recuperación de conidios en moscas infectadas. Las moscas que presentaron micosis, confirmada mediante observaciones realizadas utilizando un microscopio estereoscópico, permanecieron en las cámaras húmedas por un periodo $\geq 15$ días, tiempo requerido para el proceso de esporulación por cada cepa. Por cada cepa y concentración evaluada, se tomaron aleatoriamente cinco moscas muertas que se colocaron en forma individual en viales de vidrio con $2 \mathrm{~mL}$ de agua destilada + $0.01 \%$ de glicerina, posteriormente cada tubo fue homogenizado en un vortex durante 10 minutos para desprender los conidios del cuerpo del insecto. De esta solución acuosa se realizaron las diluciones de $10^{-1}$ a $10^{-4}$ y utilizando un hemocitómetro se cuantificó el número de conidios producidos por las moscas infectadas. Por cada concentración y por cada cepa se realizaron al menos cinco repeticiones. La esporulación fue estimada a partir de la cantidad de conidios producida por mosca infectada expresada en porcentaje. 
Análisis de datos. Antes de realizar el análisis de los datos, la mortalidad ocasionada por cada cepa fue corregida a partir de la mortalidad natural que se registró para las moscas del testigo, utilizando la formula de Abbott (1925), considerando que los bioensayos cumplieran con lo requisitos estadísticos establecidos para este tipo de estudios por Ibarra \& Federeci (1987). Los valores de tiempo letal medio $\left(\mathrm{TL}_{50}\right)$ se estimaron por máxima verosimilitud utilizando una distribución normal y posteriormente fueron comparados para determinar si había o no diferencias entre ellos. Para estimar qué cepa fue la más patogénica, se compararon los valores de intervalo de confianza (limites fiduciales) al 95\% que se determinaron para cada una de ellas. Los datos de esporulación del hongo sobre los cadáveres expresados en porcentajes fueron transformados de acuerdo a la formula arc. sen $\sqrt{\mathrm{x}}$ (Little \& Jackson 1989) y posteriormente fueron comparados entre sí para determinar si había o no diferencias entre ellos; además, se hizo una correlación entre la cantidad de conidios producidos por las moscas infectadas y la concentración del inoculo aplicada. Todos los análisis fueron realizados con el programa S-PLUS Life Data Analysis JMP versión 4 (SPLIDA 2005).

\section{RESULTADOS}

Patogenicidad hacia adultos. Con base en los resultados obtenidos, las tres cepas de hongos fueron patogénicas a $A$. obliqua, registrándose una alta mortalidad en los adultos tratados. Debido a que no hubo diferencias en la mortalidad entre hembras y machos, los valores para los análisis fueron considerados como mortalidad total. La cepa que mayor mortalidad ocasionó fue la $\mathrm{Bb}_{26}$, seguida por la cepa $\mathrm{Bb}_{\mathrm{JLSV}} \mathrm{y}$ por último fue la cepa $\mathrm{Ma}_{\mathrm{CENGICAÑA}}$ (Cuadro 2). Sin embargo, las diferencias entre los valores de mortalidad no fueron significativas $(P \geq 0.05)$.

Cuadro 2. Mortalidad de adultos de A. obliqua tratados con tres cepas de hongos entomopatógenos en condiciones de laboratorio. ( $N=90$ adultos/cepa).

\begin{tabular}{lcc}
\hline Cepa & Dosis $($ Conidios ml-1) & Mortalidad (\%)* \\
\hline $\mathrm{Bb}_{26}$ & $1.52 \times 10^{8}$ & $99.8 \mathrm{a}$ \\
$\mathrm{Bb}_{\text {JLSV }}$ & $1.76 \times 10^{8}$ & $93.5 \mathrm{a}$ \\
$\mathrm{Ma}_{\text {CENGICAÑA }}$ & $1.50 \times 10^{8}$ & $89.8 \mathrm{a}$ \\
\hline
\end{tabular}

* Corregida por la fórmula de Abbott.

Tiempo letal medio $\left(\mathbf{T L}_{\mathbf{5 0}}\right)$. La cepa que menor tiempo requirió para matar al $50 \%$ de los insectos tratados fue la $\mathrm{Ma}_{\mathrm{CENGICAÑA}}$, y la que requirió mayor tiempo fue la $\mathrm{Bb}_{\mathrm{JLSV}}$ (Cuadro 3). Las diferencias observadas entre los valores de tiempo letal medio $\left(\mathrm{TL}_{50}\right)$ fueron significativas entre cepas, pero no entre sexos por cada cepa $(P \geq 0.05)$. 
Hernández et al.: Susceptibilidad de A. obliqua a tres cepas de hongos entomopatógenos

Cuadro 3. Tiempo letal medio $\left(\mathrm{TL}_{50}\right)$ para adultos de $A$. obliqua expuestos a tres hongos entomopatógenos. $(N=90$ adultos/cepa).

\begin{tabular}{lccc}
\hline Cepa & Hospedero original & Orden: Familia & TL $_{\mathbf{5 0}}$ (días) \\
\hline $\mathrm{Ma}_{\mathrm{CENGICAÑA}}$ & Aeneolamia postica & Homoptera: Cercopidae & 3.9 \\
$\mathrm{Bb}_{26}$ & Hypothenemus hampei & Coleoptera: Scolytidae & 5.3 \\
$\mathrm{Bb}_{\mathrm{JLSV}}$ & H. hampei & Coleoptera: Scolytidae & 6.4 \\
\hline
\end{tabular}

Concentración letal media $\left(\mathbf{C L}_{\mathbf{5 0}}\right)$. En este estudio la cepa más patogénica fue la $\mathrm{Bb}_{26}$ y la menos patogénica fue la cepa $\mathrm{Ma}_{\text {CENGICAÑa. Este hecho indicó que con la }}$ cepa más patogénica, se requiere menor cantidad de inoculo (conidios) para infectar y matar al $50 \%$ de la población tratada bajo las condiciones descritas para este estudio (Cuadro 4). Las diferencias entre la mortalidad de hembras y machos tratados con cada cepa de hongo no fueron significativas.

Cuadro 4. Concentración letal media $\left(\mathrm{CL}_{50}\right)$ estimada en conidios $\mathrm{ml}^{-1}$ para tres cepas de hongos entomopatogenos sobre adultos de A. obliqua. $(N=720$ adultos/cada cepa de hongo).

\begin{tabular}{lccc}
\hline Cepa & $\begin{array}{c}\mathbf{C L}_{\mathbf{5 0}}(\text { Conidios ml-1) } \\
\text { e intervalo de confianza al 95\% } *\end{array}$ & $\chi^{\mathbf{2}}$ & $\begin{array}{c}\text { Ecuación de la línea } \\
\text { de regresión }\end{array}$ \\
\hline $\mathrm{Bb}_{26}$ & $3.8(1.97-6.99) \times 10^{6} \mathrm{a}$ & 6.44 & $\mathrm{y}=1.42 \mathrm{x}+11.8$ \\
$\mathrm{Ma}_{\text {CENGICAÑA }}$ & $4.8(3.75-6.30) \times 10^{6} \mathrm{ab}$ & 4.94 & $\mathrm{y}=0.46 \mathrm{x}+3.74$ \\
$\mathrm{Bb}_{\mathrm{JLSV}}$ & $8.8(5.45-10.46) \times 10^{6} \mathrm{~b}$ & 4.48 & $\mathrm{y}=0.99 \mathrm{x}+7.50$ \\
\hline
\end{tabular}

* Valores con la misma letra son estadísticamente iguales de acuerdo a sus intervalos de confianza (limites fiduciales) al $95 \%$.

Producción y recuperación de conidios en moscas infectadas. La cepa que mayor esporulación expresada en porcentaje presentó fue $\mathrm{Bb}_{26}$ con $77.9 \%$ cuando los insectos fueron tratados con la mayor concentración (1.0\%), seguida por la cepa Ma $_{\text {CENGICAÑA y }} \mathrm{Bb}_{\text {JLSV }}$ con 73.8 y $72.6 \%$, respectivamente (Fig. 1). Sin embargo, la cepa $\mathrm{Ma}_{\text {CENGICAÑa }}$ con la concentración de $0.001 \%$, provocó mayor mortalidad $(12.2 \%)$ comparada con las otras dos cepas, que generaron una mortalidad del $8.5 \%$ a la misma concentración (Fig. 1).

De acuerdo con los resultados obtenidos, el promedio general de producción de esporas para las tres cepas fue de $45.6 \%$, pero la cepa $\mathrm{Bb}_{26}$ registró la mayor producción (48.4\%) con respecto a la media total, lo cual indicó que esta cepa fue la 


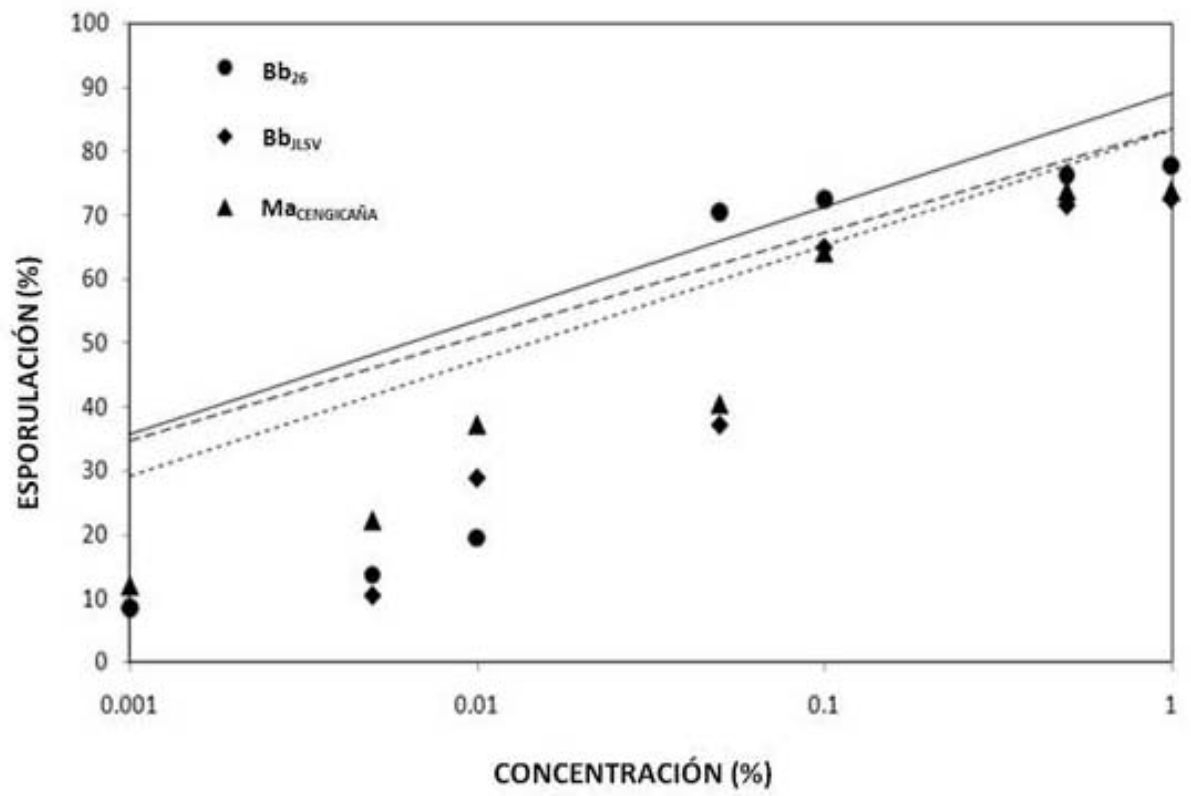

Figura 1. Tendencia de la esporulación para dos cepas de B. bassiana y una de $M$. anisopliae en adultos muertos e infectados de A. obliqua.

más patogénica de las tres, seguida por la cepa $\mathrm{Ma}_{\text {CENGICAÑA }}$ con $46.3 \%$ y la $\mathrm{Bb}_{\mathrm{JLSV}}$ con $42.0 \%$ cuyos valores fueron cercanos a la media general. Este hecho indico que las tres cepas tienen una actividad biológica similar (Fig. 1).

La esporulación de los hongos sobre los cadáveres infectados registró una correlación positiva entre la concentración aplicada y la producción de conidios en los insectos infectados $\left(\mathrm{R}^{2}=0.91, \mathrm{Y}=1.63+0.14 \mathrm{x}\right)$. Es decir que a medida que se incrementó la concentración del inoculo de cada cepa, la esporulación registrada en los insectos infectados fue mayor (Fig. 1).

\section{DISCUSIÓN}

En este estudio se observó que la infección y muerte ocasionada por las tres cepas de hongos en adultos de A. obliqua fue muy similar a los porcentajes que se han reportado para otras cepas evaluadas contra distintas especies de tefrítidos. Para adultos de $C$. capitata tratados con conidios de $M$. anisopliae, se registró entre el 78.5 y el $100 \%$ de mortalidad (Espín-García et al. 1989). Con cepas de esta misma especie de hongo, para hembras de A. ludens se observó de 86.5 a $97.3 \%$ de infección (Campos 2000) y con cepas de $M$. anisopliae, la infección fue de entre el 37.9 y el $98.7 \%$ en ambos sexos (Lezama-Gutiérrez et al. 2000). Sin embargo, la patogenicidad r para varias cepas de 
B. bassiana contra adultos de A. ludens y C. capitata, provoco un mayor rango de mortalidad (20 y el 98.7\%) (De la Rosa et al. 2002, Muñoz et al. 2009). Estos resultados evidencian que la patogenicidad de las cepas es muy variable y tiene una relación directa con la especie del insecto hospedero.

La fase adulta de las moscas de la fruta no siempre es susceptible a la infección por hongos entomopatógenos y en algunos casos la infección no se expresa como se ha reportado para otras especies de insectos que son más susceptibles. Cabe mencionar que el desarrollo del hongo sobre los insectos infectados se lleva a cabo solo cuando la interacción ocurre bajo condiciones óptimas de humedad para promover el crecimiento y la esporulación, que es cuando se produce el conidio asexual infectivo sobre los cadáveres infectados (Hajek \& St. Leger 1994, Paterson et al. 1994, Lecuona et al. 1995). Los insectos infectados al inicio de la esporulación adquieren una coloración acorde de la especie del hongo con que fue tratado (Roberts 1966, Duriez et al. 1981, McCoy et al. 1988). En el caso de A. obliqua, la esporulación observada en los insectos infectados fue típica de las cepas evaluadas, y en condiciones naturales, tiene la ventaja de que una vez que los insectos han esporulado, los conidios del patógeno se diseminan y prevalecen en el ambiente ocasionando nuevas infecciones, si las condiciones de humedad y temperatura son las óptimas (Bidochka \& Khachatourians 1994, Fargues et al. 1997).

A pesar de que las cepas evaluadas en este estudio no se obtuvieron de moscas infectadas bajo condiciones naturales, podemos afirmar con base en la infección observada y la cantidad de conidios producidos en los adultos tratados, que son patogénicas para tefrítidos. Sin embargo, no debemos olvidar que el origen de la cepa del patógeno y del hospedero, están estrechamente relacionados con su capacidad infectiva. A este respecto, Espín-García et al. (1989), reportaron que los valores de $\mathrm{TL}_{50}$ variaron de 11 a 14 días para adultos de $C$. capitata después de haber sido tratados con diversas cepas de B. bassiana aisladas en territorio Brasileño. Para cepas de la misma especie de hongo aisladas en diferentes hospederos de varias regiones de México y evaluadas bajo condiciones similares a las descritas en este estudio contra hembras de $C$. capitata, el $\mathrm{TL}_{50}$ varió de 3.83 a 17.64 días (Muñoz et al. 2009). Por lo tanto, los valores de $\mathrm{TL}_{50}$ determinados para adultos de A. obliqua tienen similitud con valores de $\mathrm{TL}_{50}$ reportados previamente para $A$. ludens por otros autores (De la Rosa et al. 2002).

De acuerdo con los valores de $\mathrm{TL}_{50}$ que se obtuvieron, estas cepas se pueden clasificar en dos grupos; el primer grupo corresponde a las cepas $\mathrm{Bb}_{26}$ y $\mathrm{Ma}_{\text {CENGICAÑA, }}$ ya que sus intervalos de confianza se traslapan entre sí, y el segundo grupo, la cepa $\mathrm{Ma}_{\text {CENGICAÑa }}$ y $\mathrm{Bb}_{\mathrm{JLSV}}$, dado que también se traslapan sus intervalos de confianza al 95\% y son estadísticamente iguales entre sí pero diferente a la primera cepa. La cepa $\mathrm{Ma}_{\text {CENGICAÑA }}$ queda comprendida en los dos grupos ya que sus intervalos de confianza al 95\% se traslapan con ambos grupos (Cuadro 4). La única diferencia entre las cepas 
evaluadas fue con relación al $\mathrm{TL}_{50}$, por lo que con la $\mathrm{CL}_{50}$, se puede predecir que cualquier concentración de estas cepas que se utilice para tratar adultos de A. obliqua, los resultados serán similares. Los intervalos de confianza tienen su aplicación en la comparación de la $\mathrm{CL}_{50}$ de dos o más productos o poblaciones de insectos expuestas a un determinado estímulo (tóxico, patógeno, etc.) (Lagunes 1991). Si el intervalo de confianza (95\%) inferior o superior al de la $\mathrm{CL}_{50}$ de las cepas evaluadas se encuentra entre los rangos de valores de los intervalos de confianza de las $\mathrm{CL}_{50}$ 's de alguna de ellas, entonces estas cepas estadísticamente son iguales (con un nivel de 95\% de confianza) considerando la respuesta al patógeno evaluado.

Los resultados de la $\mathrm{CL}_{50}$ obtenidos para A. obliqua son comparables con los obtenidos para A. ludens, tratadas con tres cepas de $M$. anisopliae, obteniéndose una $\mathrm{CL}_{50}$ de 4.38 x $10^{6}$ conidios $\mathrm{ml}^{-1}$ para la cepa $\mathrm{Ma}_{3}$, de 6.27 x $10^{6}$ conidios $\mathrm{ml}^{-1}$ para la cepa $\mathrm{Ma}_{5}$, y de 9.47 x $10^{6}$ conidios ml-1 para la cepa nativa $\mathrm{Ma}_{\mathrm{NAT}}$ (colectada en el Soconusco, Chiapas, México) y tres cepas de B. bassiana con una $\mathrm{CL}_{50}$ equivalente a $5.13 \times 10^{5}$ conidios $\mathrm{ml}^{-1}$ para la cepa $\mathrm{Bb}_{16}$, de $3.12 \times 10^{6}$ conidios ml${ }^{1}$ para la cepa $\mathrm{Bb}_{24}$, y de $9.07 \times 10^{6}$ conidios $\mathrm{ml}^{-1}$ para la cepa $\mathrm{Bb}_{26}$ (Campos 2000, De la Rosa et al. 2002).

La actividad biológica de ambas especies de hongos entomopatógenos sobre insectos se ha demostrado a través de diversos métodos de tratamiento (contacto, ingestión, transmisión, etc.), considerando además la interacción de algunos factores bióticos y abióticos como la temperatura, humedad relativa, tipo de suelo, origen de la cepa del hongo, edad y especie de mosca de la fruta (Dimbi et al. 2003, ArévaloNiño et al. 2006, Herrera \& Mata 2006, Quesada-Moraga et al. 2006, Toledo et al. 2007a). Este hecho indica que las cepas con actividad biológica tienen potencial para ser utilizadas como un elemento más de mortalidad para el manejo de dichas plagas. Por lo que el control microbiano de moscas de la fruta utilizando hongos entomopatógenos podría hacerse a través de varias vías: 1) aplicados directamente contra las larvas (incorporándolo al suelo o asperjándolo sobre la superficie cuando las larvas se están enterrando para pupar); 2) dirigido hacia adultos jóvenes, 3) liberando insectos estériles como vectores, previamente tratados con conidios, o 4) utilizando estaciones cebo (atrayente + conidios).

El mango es infestado tanto por A. ludens como por A. obliqua, este tipo de interacción hace que sea necesario evaluar las cepas de microorganismos entomopatógenos contra cada especie de mosca de la fruta, y posteriormente evaluar bajo condiciones de campo para determinar la concentración óptima contra la especie de mayor tolerancia. Si consideramos estos aspectos, se lograra mayor eficacia cuando se utilicen en el manejo de dichas plagas. Por otro lado, debemos de considerar que los insecticidas sintéticos aplicados ya sea asperjados o como cebos tóxicos para el control de moscas de la fruta, generan problemas de contaminación y efectos adversos sobre organismos no blanco (Troestchler 1984, Croft 1990, Purcell \& Shcroeder 1996), por 
lo que cada vez hay mayor interés por el aislamiento y caracterización de nuevas cepas de hongos entomopatógenos para identificar aquellas con actividad biológica (Sookar et al. 2008). Las tres cepas evaluadas resultaron patógenas a adultos de A. obliqua, por lo que deben ser evaluadas en condiciones de campo, ya sea contra adultos emergiendo a través del suelo tratado y/o mediante dispositivos que liberen conidios (e.g. alimentocebo, propuesto por Quesada-Moraga et al. 2008). El trabajo hecho por Dimbi et al. (2003) apoya esta posibilidad ya que demostraron que las moscas son infectadas cuando caminan sobre superficies tratadas con conidios del hongo. Aunque la transmisión horizontal tiene la ventaja de infectar a las moscas que se localizan en sitios con hospederos alternos o refugios de difícil acceso (Toledo et al. 2007b, QuesadaMoraga et al. 2008). Actualmente, se están realizando algunas investigaciones considerando la interacción del patógeno con factores abióticos para determinar su actividad biológica bajo dichas condiciones. Por lo tanto, el uso de insectos estériles como transmisores de conidios de ambas especies de hongos entomopatógenos, ofrece potencial para utilizarlos de manera más eficaz (Toledo et al. 2007b, Quesada-Moraga et al. 2008). Una vez que se determinó la actividad biológica de estas cepas bajo las condiciones indicadas en este estudio, se sugiere evaluarlas a nivel de campo para desarrollar la forma o el mecanismo de aplicación para incorporarlos como un elemento más de mortalidad en el manejo de esta plaga, principalmente en agroecosistemas que cuentan con certificación de sistemas de producción orgánica. Sin olvidar que es necesario considerar el desarrollo del insecto plaga y la diversidad del ambiente y el sistema de producción del cultivo en donde se utilicen dado que siempre será necesario cumplir con los requisitos de sanidad exigidos por los diferentes mercados compradores de fruta.

AGRADECIMIENTOS. A dos revisores anónimos y a J. Rull, por sus valiosas observaciones y sugerencias para mejorar la calidad del manuscrito. A Javier Valle por su asesoría con los análisis estadísticos. A Gustavo Rodas por su apoyo técnico. Al laboratorio de cría del Programa Moscafrut (SAGARPA-IICA) por el material biológico proporcionado.

\section{LITERATURA CITADA}

Abbott, W. S. 1925. A method of computing the effectiveness of an insecticide. Journal of Economic Entomology, 18: 265-267.

Aluja, M. 1994. Bionomics and management of Anastrepha. Annual Review of Entomology, 39: 155173.

Arévalo-Niño, K., A Saucedo-Casados, L. Morales-Ramos, E. Huerta-Alemán, \& C. Solís-Rojas. 2006. Implementación de la cría masiva de la mosca mexicana de la fruta Anastrepha ludens (Loew) (Diptera: Tephritidae), para la selección de hongos entomopatógenos. Pp. 410-413. In: Memorias del XXIX Congreso Nacional de Control Biológico. Sociedad Mexicana de Control Biológico. Manzanillo, Colima, México.

Artiaga-López, T., E. Hernández, J. Domínguez-Gordillo, D. S. Moreno, \& D. Orozco-Dávila. 2004. Mass-production of Anastrepha obliqua at the Moscafrut Fruit Fly Facility, Mexico. Pp. 389- 
392. In: B. N. Barnes (ed.). Proceedings of the Sixth International Symposium on Fruit Flies of Economic Importance. Irene, South Africa, Isteg Scientific Publications.

Bidochka, M. J., \& G. G. Khachatourians. 1994. Basic proteases of entomopathogenic fungi differ in their properties to insect cuticle. Journal of Invertebrate Pathology, 64: 26-32.

Campos, C., S. E. 2000. Selección de cepas de Metarhizium anisopliae (Mestch) Sorokin virulentas a la mosca mexicana de la fruta Anastrepha ludens (Loew) en condiciones de laboratorio. Tesis de Licenciatura. Facultad de Ciencias Agrícolas. Universidad Autónoma de Chiapas. Huehuetán, Chiapas, México. 60 pp.

Croft, B. A. 1990. Arthropod Biological Control Agents and Pesticides. Wiley, New York.

De la Rosa, W., F. L. López, \& P. Liedo. 2002. Beauveria bassiana as a pathogen of the Mexican fruit fly (Diptera: Tephritidae) under laboratory conditions. Journal of Economic Entomology, 95: 36-43.

Dimbi, S., N. K. Maniania, A. L. Slawomir, \& J. K. Mueke. 2003. Pathogenicity of Metarhizium anisopliae (Metsch.) Sorokin and Beauveria bassiana (Balsamo) Vuillemin, to three adult fruit fly species: Ceratitis capitata (Weidemann), C. rosa var. fasciventris Karsch and C. cosyra (Walker) (Diptera: Tephritidae). Mycopathologia, 156: 375-382.

Duriez, T. V., J. Fargues, P. H. Robert, \& R. Popeye. 1981. Etude enzimatique compare de champignons entomopathogénes des generes Beauveria et Metarhizium. Mycopathologia, 75: 109-126.

Espín-García, A. T., H. M. L. de Souza, C. L. Messías \& A. E. Piedrabuena. 1989. Patogenicidade de Metarhizium anisopliae nas diferentes fases de desenvolvimento de Ceratitis capitata (Wied.) (Diptera, Tephritidae). Revista Brasileira de Entomologia, 33: 17-23.

Fargues, J., A. Ouedraogo, M. S. Goettel, \& C. J. Lomer. 1997. Effect of temperature, humidity and inoculation method on susceptibility of Schistocerca gregaria to Metarhizium flovoride. Biocontrol Science and Technology, 7: 345-356.

García, A. T. E., C. L. Messias, H. M. L. de Souza, \& A. E. Piedrabuena. 1984. Patogenicidade de Metarhizium anisopliae var. anisopliae a Ceratitis capitata (Wiedemann) (Diptera: Tephritidae). Revista Brasileira de Entomologia, 28: 421-424.

Hajek, A. E., \& R. J. St. Leger. 1994. Interactions between fungal pathogens and insects host. Annual Review of Entomology, 39: 293-322.

Herrera, M. J., \& G. X. Mata. 2006. Evaluación de la patogenicidad de cuatro géneros de hongos entomopatógenos para el control de Ceratitis capitata y Anastrepha obliqua bajo condiciones de laboratorio. Pp. 296-297. In: Memorias del XXIX Congreso Nacional de Control Biológico. Manzanillo, Col., México.

Ibarra, J. E., \& B. A. Federici. 1987. An alternative bioassay employing neonate larvae for determining the toxicity of suspended particles to mosquitoes. Journal of the American Mosquito Control Association, 3: 187-192.

Jiménez, J. A. 1992. Patogenicidad de diferentes aislamientos de Beauveria bassiana sobre la broca del café. Cenicafe. 43: 84-98.

Lagunes, T. A. 1991. Notas del curso de toxicología y manejo de insecticidas (Documento de trabajo). Centro de Entomología y Acarología. Colegio de Postgraduados. Montecillo, Chapingo, México. $246 \mathrm{pp}$.

Lecuona, R., B. Papierok, \& G. Riba. 1995. Hongos entomopatógenos. Pp. 35-60. In: R. E. Lecuona (ed.). Microorganismos patógenos empleados en el control microbiano de insectos plaga. Buenos Aires, Argentina. 338 pp.

Lezama-Gutiérrez, R., A. Trujillo de la Luz, J. Molina-Ochoa, O. Rebolledo-Domínguez, A. R. Pescador, M. Lopez-Edwars, \& M. Aluja. 2000. Virulence of Metarhizium anisopliae (Deuteromycotina: Hyphomycetes) on Anastrepha ludens (Diptera: Tephritidae) laboratory and field trials. Journal of Economic Entomology, 93: 1080-1084. 
Little, T. M., \& H. Jackson. 1989. Métodos estadísticos para la investigación en la agricultura. Ed. Trillas. México, D. F. 246 pp.

Magaña, C., P. Hernández-Crespo, F. Otergo, \& P. Castañera. 2007. Resistance to malation in field populations of Ceratitis capitata. Journal of Economic Entomology, 100: 1836-1843.

McCoy, C. W., R. A. Samson, \& D. G. Boucias. 1988. Entomogenous fungi. Pp. 151-236. In: C. M. Ignoffo and N. B. Mandava (eds.). Handbook of Natural Pesticidas, Microbial Insecticidas. Vol. V, Part A. Entomogenous Protozoa and Fungi. Boca Raton, Fl.

Muñoz, J. A., W. de la Rosa, \& J. Toledo. 2009. Mortalidad en Ceratitis capitata (Wiedemann) (Diptera: Tephritidae) por diversas cepas de Beauveria bassiana (Bals.) Vuillemin, en condiciones de laboratorio. Acta Zoológica Mexicana, 25: 609-624.

Paterson, I. C., A. K. Charnley, R. M. Cooper, \& J. M. Clarkson. 1994. Specific induction of a cuticle-degrading protease of the insect pathogenic fungus Metarhizium anisopliae. Microbiology, 140: 185-189.

Purcell, M. F., \& W. J. Schroeder. 1996. Effect of silwet L-77 and diazinon on three tephritide fruit flies (Diptera: Tephritideae) and associated endoparasitoids. Journal of Economic Entomology, 89: 1566-1570.

Quesada-Moraga, E., A. Ruíz-García, \& C. Santiago-Álvarez. 2006. Laboratory evaluation of entomopathogenic fungi Beauveria bassiana and Metarhizium anisopliae against puparias and adults of Ceratitis capitata (Diptera: Tephritidae). Journal of Economic Entomology, 99: 1955-1966.

Quesada-Moraga, E., I. Martín-Carballo, I. Garrido-Jurado, \& C. Santiago-Álvarez. 2008. Horizontal transmission of Metarhizium anisopliae among laboratory populations of Ceratitis capitata (Wiedemann) (Diptera: Tephritidae). Biological Control, 47: 115-124.

Reyes, F., J., G. Santiago M., \& P. Hernández M. 2000. The Mexican fruit fly eradication programme. Pp. 377-380. In: K. H. Tan (ed.). Area-Wide Control of Fruit Flies and Other Insect Pests. Penerbit Universiti Sains Malaysia, Penang.

Roberts, D. W. 1966. Toxins of entomogenous fungus Metarhizium anisopliae: Symptoms and detection in moribund host. Journal of Invertebrate Pathology, 8: 469-473.

Sookar, P., S. Bhagwant, \& E. A. Ouna. 2008. Isolation of entomopathogenic fungi from the soil and their pathogenicity to two fruit fly species (Diptera: Tephritidae). Journal of Applied Entomology, 132: 778-788.

[SPLIDA]. S - Plus Life Data Analysis. 2005. S - Plus Life Data Analysis. Copyright 1995 - 2005. W. Q. Meeker.

Toledo, J., P. Liedo, S. Flores, S. E. Campos, \& P. Montoya. 2007a. Potencial de los hongos entomopatógenos Beauveria bassiana y Metarhizium anisopliae para el control de moscas de la fruta. Pp. 157-168. In: V. Hernández-Ortíz (ed.), Moscas de la fruta en Latinoamérica (Díptera: Tephritidae): Diversidad, biología y manejo. Edit. S y G Editores. México, D. F.

Toledo, J., S. E. Campos, S. Flores, P. Liedo, J. F. Barrera, A. Villaseñor, \& P. Montoya. 2007b. Horizontal transmission of Beauveria bassiana in the Mexfly, Anastrepha ludens (Diptera: Tephritidae), under laboratory and field-cage conditions. Journal of Economic Entomology, 100: 291-297.

Troestchler, R. G. 1984. Effects on nontarget arthropods of Malathion bait sprays used in California to eradicate the Mediterranean fruit fly Ceratitis capitata (Wiedemann) (Diptera: Tephritidae). Environmental Entomology, 12: 1816-1822. 